\title{
A method of three-dimensional subdivision of arbitrary polyhedron by
}

\section{using pyramids}

\author{
LIU Ji-bo ${ }^{1, a^{*}}$, Wang Zhi-hong ${ }^{1, b}$, Yan Yue-guan ${ }^{2, c}$ \\ ${ }^{1}$ College of Mining Engineering, Guizhou University of Engineering Science, Guizhou, 551700, \\ China \\ ${ }^{2}$ College of Geoscience and Surveying Engineering, China University of Mining and Technology \\ (Beijing), Beijing, 100083, China \\ aljb0919@126.com, b258039289@qq.com, yyg_0720@163.com
}

\begin{abstract}
Keywords: arbitrary polyhedron, surface boundary, pyramid, three-dimensional subdivision Abstract. The key research of this paper is the algorithm that using pyramids to divide the arbitrary polyhedron. The core idea of this algorithm is as follows. Store the vertices of the outer boundary surfaces of the polyhedron anticlockwise, and store the vertices of the inner boundary surfaces clockwise. When the selected vertex is visible to the given polygon and the distance between them is the minimum, the pyramid composed of the point and the polygon is an effective subdivision. If there are more than one vertex have the same distance to the polygon, the projection of the vertex in the polygon should have the minimum distance to the center of gravity of the polygon.
\end{abstract}

\section{Introduction}

Delaunay triangulation algorithm for arbitrary polygon in two-dimensional space is very mature, Ma Xiao-hu and other scholars had put forward a fast algorithm of simple polygon Delaunay triangulation based on determination of convex-concave vertices. This algorithm is easy to implement, but only fits for simple polygons. Li Nan and others had presented an improved Delaunay triangulation algorithm for the arbitrary polygon by using directed edge, which is more efficient, and avoids a "default position" error, also improves the robustness of the algorithm. Many other scholars had also proposed correlated algorithms. On the basis of these studies, this paper proposed a method of three-dimensional subdivision of arbitrary space polyhedron by using pyramids.

\section{Definitions}

Definition 1: Boundary Surfaces. The directed surfaces surrounding arbitrary spatial region are known as regional boundary surfaces, which contain the outer boundary surfaces and the inner boundary surfaces. This article stipulates that the outer boundary surface's positive direction is the same as the normal vector point to the lateral border, and the inner boundary surface's positive direction is the same as the normal vector point to the medial border.

Definition 2: Visible. When each edge of the pyramid constituted by a known planar polygon and an outer point $\mathrm{P}$ does not intersect any other boundary polygons, then the point $\mathrm{P}$ is known as visible to the planar polygon. 
Definition 3: Distance of Vertex-Gravity Center. As shown in Figure 1, the distance from the projection point $\mathrm{H}$ that formed by the pyramid vertex $\mathrm{P}$ projecting onto the polygon $\mathrm{ABCD}$ to the center of gravity $\mathrm{O}$ of the pyramid bottom polygon, is known as Distance of Vertex-Gravity Center.

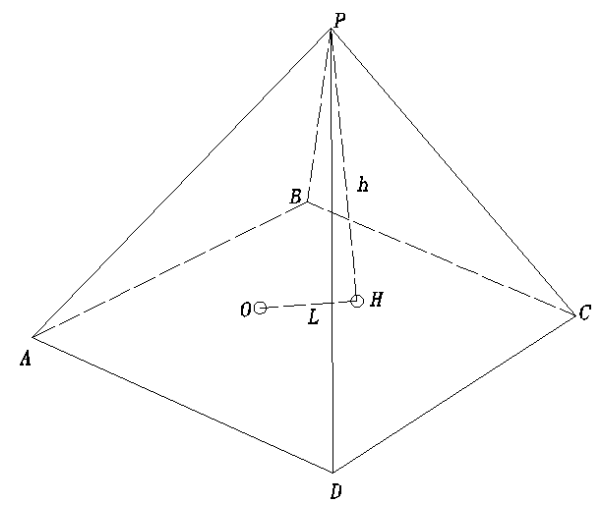

Fig. 1 Schematic diagram of the distance from point to polygon and distance between the vertex's projection and the gravity of the polygon

Property 1: The conditions of a legal three-dimensional mesh pyramid depend on: the vertex $\mathrm{P}$ is visible to the given planar polygon, and the distance between them is the minimum. When there is more than one candidate vertex, the distance from Vertex projection to Center of Gravity, i.e. $\mathrm{L}$ should be the minimum.

\section{Three-dimensional subdivision of spatial arbitrary polyhedron}

Basic ideas. Firstly, each planar polygon's vertices of outer boundary surfaces constituting spatial polyhedron $\mathrm{V}$ are stored counterclockwise (normal vectors of planar is outward), each planar polygon's vertices of inner boundary surfaces constituting spatial polyhedron are stored clockwise (normal vectors of plane is inward). In addition, each side of the spatial polyhedron has no differences toward inside or outside. The planar polygon $\pi$ with maximum edges of V's boundary surfaces will be taken out, and candidate nodes list which are visible to the polygon $\pi$ will be formed. Then get vertex $\mathrm{P}$ which can form lawful subdivision pyramid with the current polygon $\pi$ to make up another candidate nodes list. The resulting effective pyramid will be separated from spatial region V. And based on conditions whether pyramid's side faces belong to the remaining space V to modify the current space polyhedron $\mathrm{V}$. Repeat processes above until the current polyhedron $\mathrm{V}$ composed only one pyramid.

Algorithm description. Pyramid subdivision algorithm based on the directed planar polygon of arbitrary spatial polyhedron is as follows:

Step 1: Traverse the spatial polyhedral vertices, and judge whether the vertex is in the inner of the current planar polygon and calculate its visibility. If the conditions are met, then calculate the vertex's vertical distance $h$ to the planar polygon and the distance from Vertex's projection to Center of Gravity, and the vertex will be added to the list of candidate nodes.

Step 2: Use the quick sort method to sort the list of candidate nodes according to the distance $h$ from small to large. If meet the same $\mathrm{h}$, then sort according to the distance $\mathrm{L}$ from small to large.

Step 3: According to the property 1, select vertex from candidate nodes list that meets the conditions together with the given planar polygon to form an effective pyramid.

Step 4: Separate the generated pyramid from the spatial polyhedron, and adjust the boundary surfaces list of $\mathrm{V}$, that means to delete the independent surfaces from the boundary surfaces list, and 
then to add the interfaces of new generated pyramid and the spatial polyhedron to boundary surfaces list.

Step 5: Repeat step 1 to step 4, until the spatial polyhedron is subdivided completely.

\section{Data structure \& The Example}

Data structure. A good data structure design is the prerequisite for efficient algorithms. The following data structures have been defined in this article.

//Define structure VERTEX to record coordinates information

typedefstruct tagVERTEX\{

double $\mathrm{x}$; //x-coordinate

double $\mathrm{y} ; / / \mathrm{y}$-coordinate

double $\mathrm{z}$; //z-coordinate

\}VERTEX;

//Define the polygon information

typedefstruct tagPLANE\{

int $\mathrm{n}$; // the number of polygon edges

int Flag;//the flag of the polygon, if the outer boundary surface, Flag equals 1, if

the inner boundary surface, Flag equals -1

CArray $<$ VERTEX,VERTEX> PVertices;// deposit polygon vertex coordinates

VERTEX CenterPoint;// deposit coordinates of the gravity center of the polygon

\}PLANE;

//Define the pyramid information

typedefstruct tagPYRAMID \{

int SurfaceNum; //the number of the surfaces of the pyramid

CArray $<$ PLANE *, PLANE *> Surfaces; //the information of the surfaces

double $\mathrm{h}$; // vertical distance of the vertex to the polygon

double $\mathrm{L}$; //the distance between the projection point of the vertex and the gravity of the polygon

\}PYRAMID;

The Example. The hexahedron V, which outer boundary surfaces are composed of polygon ADHE, DCGH, CBFG, AEFB, ABCD and EHGF. As shown in Figure 2.

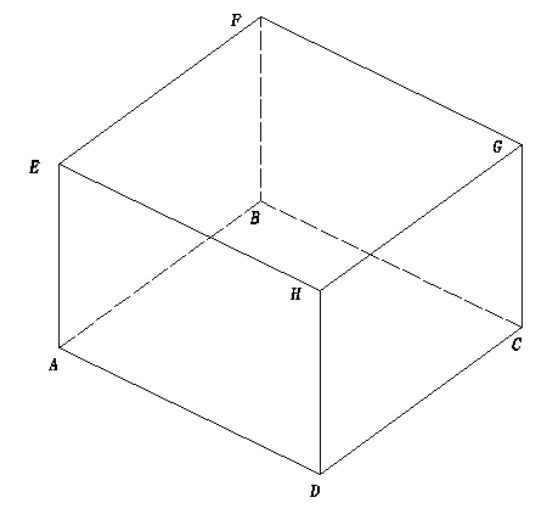

Fig. 2 Schematic diagram of the hexahedral

The first subdivision is that the polygon ADHE with the maximum edges in the list of the boundary surfaces is selected. The vertices which are visible to the polygon are B, C, G and F. According to the symmetry, all of them meet the requirements, and have the same value $h$ and L. So 
take the vertex F randomly to form the pyramid with the polygon ADHE. As shown in Figure 3(1). Rebuild the space V, and modify the boundary surfaces of residual space. As shown in Figure 3(2).



(1)

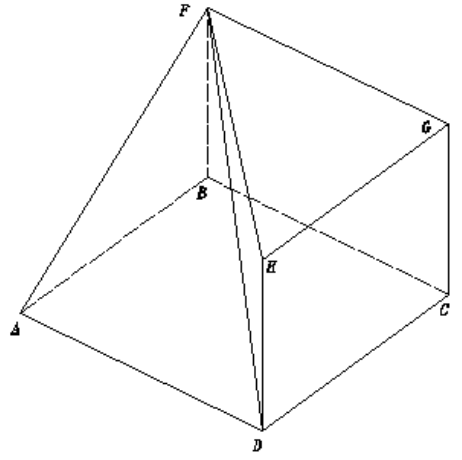

Fig. 3 Schematic diagram of the first subdivision of the hexahedral V
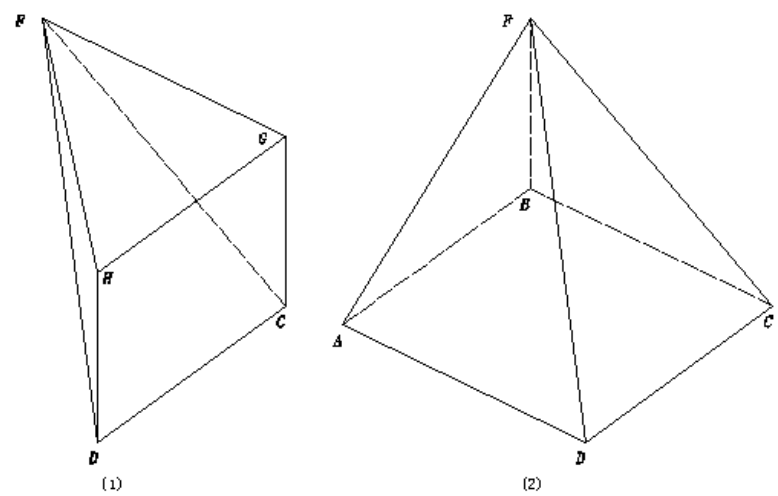

Fig. 4 Schematic diagram of the second subdivision of the hexahedral V

The second subdivision is that the polygon DCGH with the maximum edges in the list of the boundary surfaces is selected. The vertex which is visible to the polygon is F only. So take the vertex $\mathrm{F}$ to form the pyramid with the polygon DCGH. As shown in Figure 4(1). Rebuild the space $\mathrm{V}$, and modify the boundary surfaces of residual space. As shown in Figure 4(2).

The third subdivision is that the polygon ABCD with the maximum edges in the list of the boundary surfaces is selected. The vertex which is visible to the polygon is F only. So take the vertex $F$ to form the pyramid with the polygon ABCD. As shown in Figure 4(2).

So for, the subdivision of the space hexahedral V has been completed.

\section{Conclusions}

When building three-dimensional geological modeling, the spatial region always should be subdivided in order to express the three-dimensional geological modeling accurately. Based on the directed boundary surfaces, the paper achieved subdivision of the arbitrary space regions by pyramids. The algorithm is simple and efficient, which is also applicable for the conditions that there have isolated islands in the polyhedron. 


\section{Acknowledgements}

Science and technology cooperation project of Guizhou provincial science and Technology Department (Qian-Ke-He LH Word [2016] 7045); High level talent science research project of Guizhou University of Engineering Science (Yuan-Ke-He Word G2015005); National Natural Science Foundation of China (No.51404272)

\section{References}

[1] Ma Xiao-hu, Pan Zhi-geng, Shi Jiao-ying, Delaunay Triangulation of Simple Polygon Based on Determination of Convex-Concave Vertices, Journal of Computer Aided Design and Computer Graphics. 1999, 11(1):1-3

[2] Li Nan, Li Yuan, Wu Xin-cai, An Improved Delaunay Triangulation Algorithm for the Arbitrary Polygon, Microcomputer Information. 2009, 25(7):202-204

[3] Li Wei-qing, Peng Qun-sheng, A General and Fast Triangulation Algorithm, Journal of Computer-Aided Design \& Computer Graphics. 2001, 13(9):769-773

[4] Ding Yong-xiang, Xia Ju-chen, Wang Ying, Delaunay Triangulation of Arbitrary Polygons, Chinese J.Computers. 1994,17(4):270-275

[5] Chen Yong-fu, Zhang Hua, Chen Xing, Triangular Mesh Division of Arbitrary surfaces, Journal of Computer-Aided Design \& Computer Graphics. 1997, 9(5):396-401

[6] Yang Lei, Wu Tao, Robust Supplement to a Delaunay Triangulation Algorithm on 2D Arbitrary Polygon, Journal of Image and Graphics. 2000, 5(4):323-326 